\title{
Impacto del seguimiento farmacoterapéutico en pacientes adultos mayores con hipertensión arterial del Centro de Atención Primaria II Oscar Fernández Dávila
}

Pharmacotherapeutic Follow-Up Impact in Elderly Patients With Arterial Hypertension at the Oscar Fernández Dávila Primary Care Center II

Impacto do acompanhamento farmacoterapêutico em idosos com hipertensão arterial no Centro de Atenção Básica Oscar Fernández Dávila II

Liseth Rosmery Arredondo Vargas ${ }^{1 \mathrm{a}} \quad$ iD https://orcid.org/0000-0002-4521-1413

Juan Carlos Efraín Cervantes Zegarra ${ }^{16}$

\section{Resumen}

Objetivo: Determinar el impacto del seguimiento farmacoterapéutico en pacientes crónicos adultos mayores con hipertensión arterial del Centro de Atención Primaria II Oscar Fernández Dávila, EsSalud-Tacna, de julio a setiembre de 2018. Material y métodos: El estudio fue cuasi-experimental, longitudinal y ambispectivo. Se hizo el seguimiento farmacoterapéutico a 38 pacientes según el método Dáder. Resultados: Se identificaron 63 resultados negativos asociados a la medicación (RNM) como inefectividad cuantitativa $(71,43 \%)$ e inseguridad no cuantitativa $(17,46 \%)$, originados por 74 problemas relacionados con medicamentos (PRM) como incumplimiento $(55,41 \%$ ) y la probabilidad de efectos adversos (16,22 \%). Se realizó la prueba no paramétrica de Wilcoxon y se halló un impacto estadísticamente significativo positivo $(p<0,05)$ al disminuir la presión arterial sistólica (PAS) y la presión arterial diastólica (PAD). Conclusiones: Se encontró predominio de los RNM de inefectividad cuantitativa e inseguridad no cuantitativa. Se encontró predominio del PRM de incumplimiento y la probabilidad de efectos adversos. El seguimiento farmacoterapéutico generó un impacto estadísticamente significativo positivo al disminuir la PAS y la PAD.

Palabras claves: seguimiento farmacoterapéutico, método Dáder, adulto mayor, hipertensión arterial

\begin{abstract}
Objective: To determine the impact of the pharmacotherapeutic follow-up in chronic elderly patients with arterial hypertension at the Oscar Fernández Dávila Primary Care Center II, EsSalud-Tacna, from July to September 2018. Material and methods: The study was quasi-experimental, longitudinal and ambispective. Pharmacotherapeutic follow-up was done in 38 patients according to the Dader method. Results: 63 negative results associated with medication (NRM) were identified as quantitative ineffectiveness $(71.43 \%)$ and nonquantitative insecurity (17.46\%), caused by 74 medication-related problems (PRM) as non-compliance $(55.41 \%)$ and the probability of adverse effects $(16.22 \%)$. The non-parametric Wilcoxon test was performed and a statistically significant positive impact $(p<0.05)$ was found when decreasing systolic blood pressure $(S B P)$ and diastolic blood pressure (DBP). Conclusions: A predominance of the NMRs of quantitative ineffectiveness and non-quantitative insecurity was found. A predominance of the PRM of non-compliance and the probability of adverse effects was found. Pharmacotherapeutic follow-up generated a statistically significant positive impact by reducing SBP and DBP
\end{abstract}

Keywords: Pharmacotherapeutic follow-up, Dáder method, adult major and high blood pressure

\footnotetext{
${ }^{1}$ Facultad de Ciencias de la Salud, Universidad Nacional Jorge Basadre Grohmann. Tacna, Perú

${ }^{a}$ Magíster en Fisiopatología

${ }^{\mathrm{b}}$ Bachiller en Farmacia y Bioquímica
} 


\begin{abstract}
Resumo
Objetivo: Determinar o impacto do acompanhamento farmacoterapêutico em idosos crônicos com hipertensão arterial no Centro de Atenção Básica Oscar Fernández Dávila II, EsSalud-Tacna, de julho a setembro de 2018. Material e métodos: O estudo foi quase experimental, longitudinal e ambispectivo. O acompanhamento farmacoterapêutico foi realizado em 38 pacientes de acordo com o método de Dader. Resultados: 63 resultados negativos associados à medicação (NRM) foram identificados como inefetividade quantitativa $(71,43 \%)$ e insegurança não quantitativa $(17,46 \%)$, causada por 74 problemas relacionados à medicação (PRM) como incumprimento $(55,41 \%)$ e a probabilidade de efeitos adversos (16,22 \%). O teste não paramétrico de Wilcoxon foi realizado e foi encontrado impacto positivo estatisticamente significativo $(p<0,05)$ na redução da pressão arterial sistólica (PAS) e diastólica (PAD). Conclusões: Encontrou-se predomínio dos RMNs de inefetividade quantitativa e insegurança não quantitativa. Constatou-se predomínio do PRM de não conformidade e probabilidade de efeitos adversos. O acompanhamento farmacoterapêutico gerou um impacto positivo estatisticamente significativo ao reduzir a PAS e a PAD.
\end{abstract} arterial

Palavras-chave: acompanhamento farmacoterapêutico, método Dader, idoso, hipertensão

\section{Introducción}

La hipertensión arterial es conocida como "el asesino silencioso", ya que generalmente no presenta síntomas. Es la primera causa de consulta ambulatoria, en el adulto mayor, siendo la enfermedad crónica más frecuente en esta etapa de la vida según los estudios TORNASOL I y II. Esta enfermedad llega a comprometer otros órganos, desarrollar enfermedades cardiovasculares y provocar la muerte. ${ }^{1,3}$

La hipertensión arterial se hace presente a medida que envejecemos; con el paso de los años se producen cambios fisiológicos y patológicos en el paciente anciano, por lo que es de esperar que el deterioro orgánico y de los sistemas enzimáticos pueda conducir a una respuesta farmacológica alterada. En el anciano existen factores que modifican la reactividad orgánica a los fármacos tales como: variaciones farmacocinéticas y farmacodinámicas, patología múltiple asociada, polimedicación con aumento de las interacciones medicamentosas y, por tanto, mayor número de reacciones adversas y otros factores que modifican la respuesta farmacológica. Por tanto, es importante brindar atención al adulto mayor, ya que este resulta ser un paciente susceptible a sufrir resultados negativos asociados a la medicación (de aquí en adelante RNM) por causa de problemas relacionados con medicamentos (de aquí en adelante PRM). ${ }^{2}$

\section{Material y métodos}

Se realizó una investigación de tipo aplicada, diseño cuasi-experimental, longitudinal y ambispectivo, con pre y post test. La población constaba de 52 pacientes del Programa Crónico del CAP II Oscar Fernández Dávila EsSalud-Tacna, con edades entre 60 años a más, hipertensos, sin otra patología crónica. Se seleccionaron, 38 pacientes voluntarios y se les hizo firmar el consentimiento informado. El muestreo fue no probabilístico y a conveniencia.

El seguimiento farmacoterapéutico se realizó según el método Dáder, con este procedimiento, se revisaron las fichas de control de los pacientes del programa que cumplieron los criterios de elegibilidad e iniciar el seguimiento farmacoterapéutico, el cual constaba de las siguientes fases: 1) oferta de servicio, 2) primera entrevista farmacéutica, 3) estado de situación, 4) fase de estudio, 5) fase de evaluación, 6) fase de intervención y 7) entrevista farmacéutica. ${ }^{4}$

Los datos fueron procesados en SPSS v25.0, se elaboró una matriz en Microsoft Office Excel 2013. Para el análisis estadístico, se 
realizó la prueba de normalidad, prueba no paramétrica de Wilcoxon con el fin de determinar el impacto en presión arterial.

\section{Resultados}

Se identificaron 74 PRM en total (Figura 1) y 63 RNM (Figura 2). En la (Tabla 1) se identifica 79,37\% "sobre la educación del paciente". Por otro lado, al comparar la clasificación de la PAS (Figura 3 ) y PAD (Figura
4) antes y después de la intervención farmacéutica, se observó una mejoría en el estadio de la presión arterial. Finalmente, para determinar el impacto del seguimiento farmacoterapéutico, se realizó la Prueba no paramétrica de Wilcoxon, que a un nivel de $5 \%$ de significancia, resultó estadísticamente significativo al disminuir la PAS con $p$-valor $=$ $0,000(p<0,05)$ y PAD con $p$-valor $=0,045(p<$ $0,05)$ (Tabla 2).

\section{Figura 1}

Problemas relacionados con medicamentos identificados

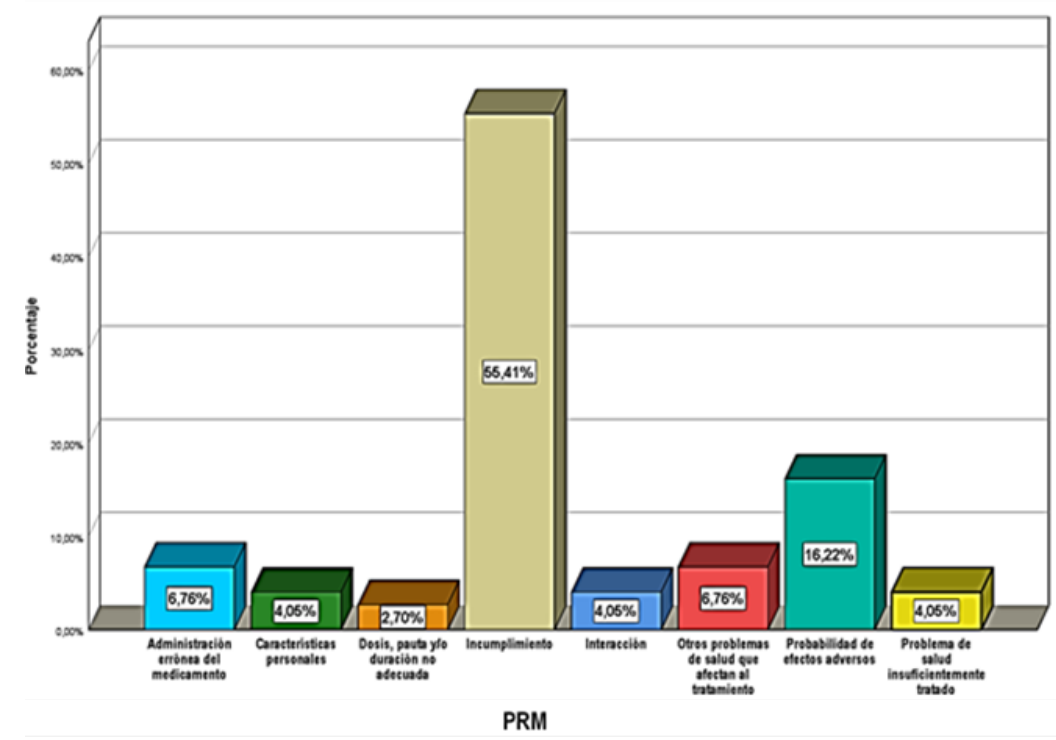

\section{Figura 2}

Resultados negativos asociados a la medicación identificados

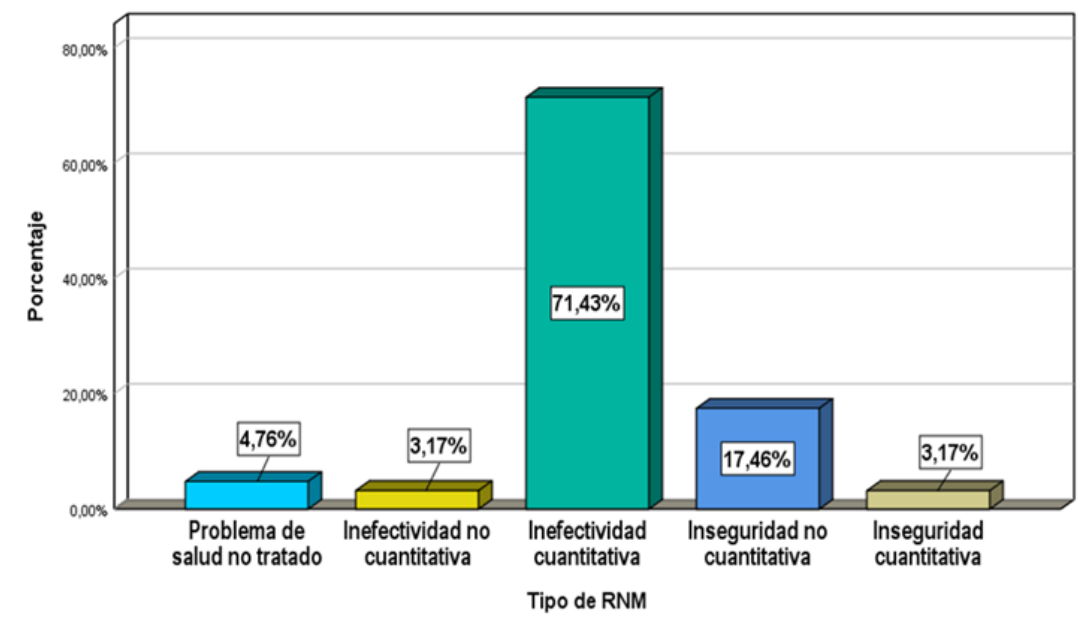




\section{Tabla 1}

Tipos de intervenciones farmacéuticas realizadas

\begin{tabular}{lcc}
\hline \multicolumn{1}{c}{ Tipo de intervención } & $n$ & $\%$ \\
\hline Sobre la cantidad de los medicamentos & 2 & 3,17 \\
Sobre la cantidad de los medicamentos & 11 & 17,46 \\
Sobre la educación del paciente & 50 & 79,37 \\
\hline Total & 63 & 100,00
\end{tabular}

\section{Figura 3}

Clasificación de la PAS antes y después de la intervención farmacéutica

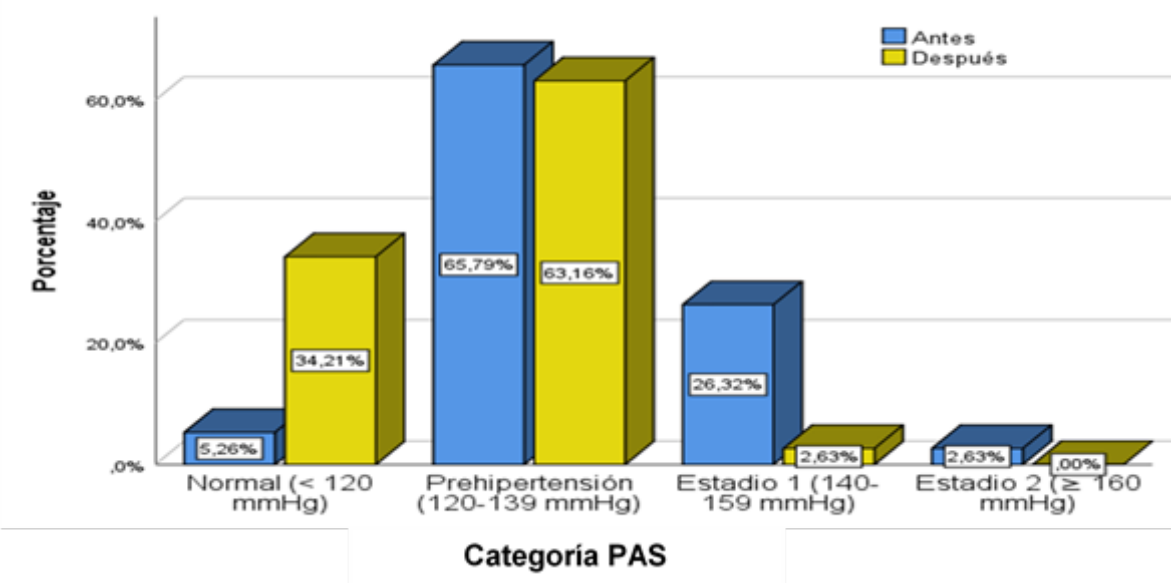

\section{Figura 4}

Clasificación de la PAD antes y después de la intervención farmacéutica

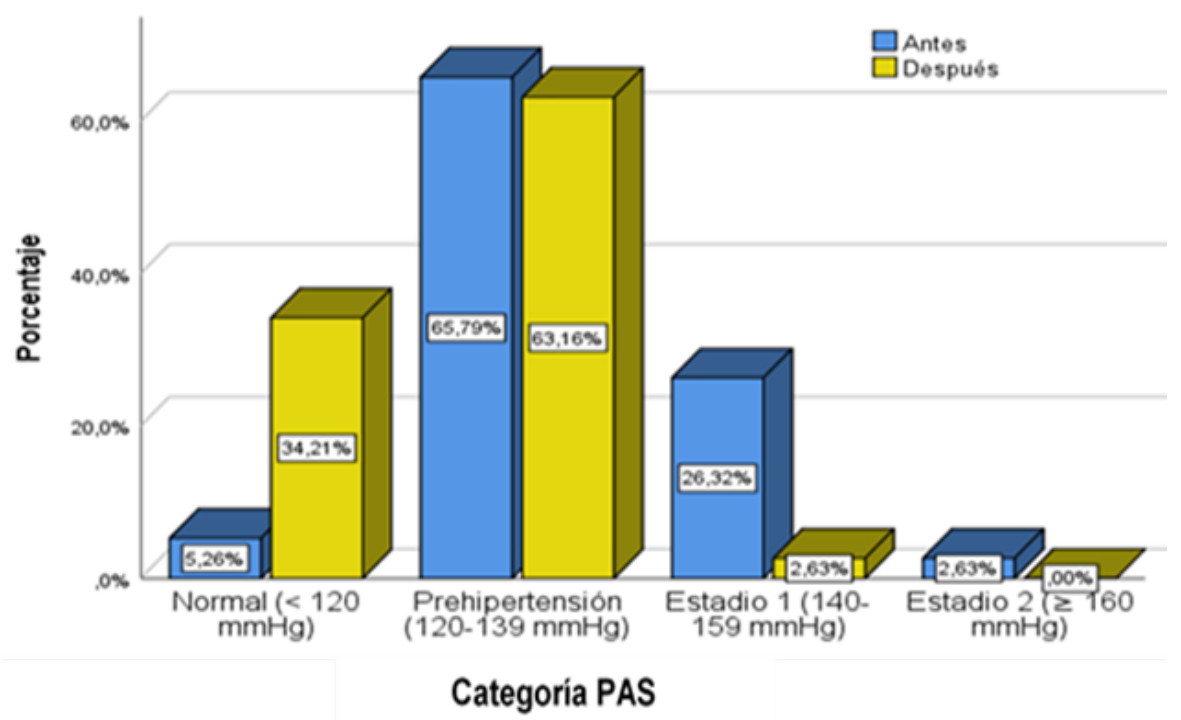




\section{Tabla 2}

Prueba no paramétrica de Wilcoxon para la PAS y PAD

\begin{tabular}{ccc}
\hline Estadístico de prueba & PAS antes - & PAD antes - PAD \\
& PAS después & después \\
\hline Z & $-4,311$ & $-2,006^{\mathrm{b}}$ \\
Sig. & 0,000 & 0,045 \\
\hline
\end{tabular}

\section{Discusión}

Al evaluar el estado de situación, se identificaron 74 PRM (Figura 1), con predominio de incumplimiento con $55,41 \%$ y probabilidad de efectos adversos con $16,22 \%$. Estos resultados concuerdan con el estudio del autor Sánchez, ${ }^{8}$ en cuyo estudio también predomina, los PRM respecto al incumplimiento con $67 \%$, seguido de la conservación inadecuada con $25 \%$. Sin embargo, difiere con el estudio de Alvarado, ${ }^{7}$ donde predomina la probabilidad de efectos adversos con $25 \%$, seguido por el incumplimiento con $23,53 \%$. La diferencia del estudio de Alvarado ${ }^{7}$ es que participaron pacientes con pluripatología, en quienes se utilizaron un mayor número de medicamentos, que aumentan el riesgo de sufrir efectos adversos. El estudio de Sánchez ${ }^{8}$ y nuestro estudio tuvieron pacientes, solo con una sola patología la hipertensión arterial crónica, por tanto, el número de medicamentos administrados fue menor, reduciendo el riesgo de interacción medicamentosa.

El incumplimiento es un problema presente en adultos mayores, por la misma edad presentan, deterioro sensorial, coordinación motora y equilibrio, baja agudeza mental y atención disminuidas, siendo unos de los problemas que causan la baja adaptación a regímenes terapéuticos o dificultan la adherencia al tratamiento. ${ }^{14,15}$

En el anciano existen factores que modifican la reactividad orgánica a los fármacos tales como: variaciones farmacocinéticas y farmacodinámicas, patología múltiple asociada, polimedicación con aumento de las interacciones medicamentosas y por tanto mayor número de reacciones adversas y otros factores que modifican la respuesta farmacológica, el deterioro sensorial y cognitivo que pueden llevar a una administración errónea del medicamento y que finalmente conllevan a una acción insegura del medicamento. ${ }^{12}$

En cuanto a los RNM (Figura 2), se identificaron 63, predominando la inefectividad cuantitativa con $71,43 \%$, seguido por inseguridad no cuantitativa con 17,46\%. En el estudio de otro autor Cajas, ${ }^{5}$ coincide con el presente trabajo ya que también predominó la inefectividad cuantitativa $(58,8 \%$ ) y en segundo lugar la inseguridad no cuantitativa (16,7\%).

La inefectividad cuantitativa se da principalmente por el incumplimiento e interacción de medicamentos, siendo unas de las causas para que se reduzcan las dosis. ${ }^{14}$ Por otro lado, la inseguridad no cuantitativa puede presentarse independiente de la dosis en el adulto mayor. Existen factores que modifican la reactividad orgánica a los fármacos tales como: variaciones farmacocinéticas y farmacodinámicas, patología múltiple asociada, polimedicación con aumento de las interacciones medicamentosas y por tanto mayor número de reacciones adversas y otros factores que modifican la respuesta farmacológica que son: menor tamaño corporal, disminución de la función renal y hepática, menor flujo sanguíneo periférico, hipoalbuminemia, tendencia a subnutrición y una disminución de la eficacia de los mecanismos homeostáticos. ${ }^{11,12}$ 
Finalmente, al determinar el impacto del seguimiento farmacoterapéutico en la presión arterial en nuestro estudio, se obtuvo una disminución estadísticamente significativa de la PAS y PAD (Tabla 2), resultados que concuerdan con los estudios de Rodríguez, ${ }^{6}$ Alvarado $^{7}$ y Rubiños ${ }^{9}$. En relación con el cumplimiento de la medicación se debe tener en cuenta las limitaciones del anciano para cumplir con ellas: la adherencia del tratamiento, la administración correcta del medicamento facilita las condiciones para que el medicamento cumpla su función. ${ }^{10,13 .}$

Se concluye que se identificaron 63 RNM, predominando la inefectividad cuantitativa con $71,43 \%$ e inseguridad no cuantitativa con 17,46 \%. Además, predominó el PRM de incumplimiento con $55,41 \%$ y la probabilidad de efectos adversos con $16,22 \%$. La intervención farmacéutica más realizada fue sobre la educación del paciente con 79,37 \%, seguido de sobre la estrategia farmacológica con $17,46 \%$ y sobre la cantidad de medicamentos con $3,17 \%$. El seguimiento farmacoterapéutico generó un impacto estadísticamente significativo al disminuir la PAS $(p<0,05)$ y la PAD $(p<0,05)$.

Se recomienda implementar un servicio de atención farmacéutica dedicada al seguimiento farmacoterapéutico a pacientes crónicos en el CAP II Oscar Fernández Dávila EsSalud; asimismo, incluir al químico farmacéutico en la atención del Programa Crónico. El farmacéutico conseguirá evitar o minimizar los RNM al implicarse en la evaluación de los resultados del tratamiento del paciente mediante el seguimiento farmacoterapéutico. Esto con el objetivo de identificar, prevenir y resolver los posibles problemas relacionados con los medicamentos (PRM). Por otra parte, su accesibilidad y su conocimiento de la totalidad de la farmacoterapia que utiliza el paciente hacen del farmacéutico el profesional idóneo para desarrollar el seguimiento respecto a la terapia farmacológica del paciente.

\section{Referencias}

1. EsSalud. La hipertensión arterial es una enfermedad crónica que puede ocasionar la muerte. [En línea]. 2016 [fecha de acceso 3 de abril del 2018]. Disponible en: http://www.essalud.gob.pe/essalud-lahipertension-arterial-es-unaenfermedad-cronica-quepuedeocasionar-la-muerte/

2. Salazar P, Rotta A, Otiniano F. Hipertensión en el adulto mayor. Rev Med Hered. ene. 2016; 27(1):60-66. D i s p n i b e e n : http://www.scielo.org.pe/scielo.php?scrip $\mathrm{t}=\mathrm{sci}$ arttext\&pid=S 1018 130X2016000100010\&lng=es\&tlng=es.

3. Segura L, Agusti R, Ruiz E. Hipertensión arterial en el Perú según el estudio TORNASOL II. Revista Peruana de Cardiología. Abr. 2011; 37(1): 19-27. Disponible en:

http://repebis.upch.edu.pe/articulos/rpc/v 37n1/a3.pdf

4. Sabater Hernández, D.; Silva Castro, M.M.; Faus Dáder, M.J. Método Dáder: guía de seguimiento farmacoterapéutico. $3^{\circ}$ ed. Granada: GIAF, 2007. 127 p. Disponible en:

[http://hdl.handle.net/10481/33051]

5. Cajas M. Impacto de la intervención farmacéutica sobre el tratamiento para hipertensión arterial en pacientes adultos mayores de la comunidad de San Rafael Bajo del Cantón Patate - Tungurahua. Riobamba: Escuela Superior Politécnica de Chimborazo; 2014. 193 p. Disponible en:

http://dspace.espoch.edu.ec/handle/123 456789/3889

6. Rodríguez A. Seguimiento farmacoterapéutico en hipertensos. Granada: Universidad de Granada; 2014. 195 p. Disponible en: https://hera.ugr.es/tesisugr/24463644.pd f

7. Alvarado J. Aplicación y evaluación del seguimiento farmacoterapéutico en pacientes con hipertensión arterial de consulta externa del Hospital José María 
Velasco Ibarra de Tena. Riobamba: Escuela Superior Politécnica de Chimborazo; 2016. 134 p. Disponible en: http://dspace.espoch.edu.ec/handle/123 456789/5711

8. Sánchez C. Evaluación del seguimiento farmacoterapéutico en pacientes hipertensos atendidos en la botica FARMAX. Trujillo: Universidad Católica Los Ángeles de Chimbote; 2016. 69 p. D i s p o n i b I e e $n$ : http://repositorio.uladech.edu.pe/handle/ 123456789/404

9. Rubiños A. Seguimie nto farmacoterapéutico a pacientes con hipertensión arterial del Albergue Central Ignacia Rodulfo Viuda de Canevaro Lima2014. Lima: Universidad Nacional Mayor de San Marcos; 2017. 63 p. Disponible en: https://hdl.handle.net/20.500.12672/699 3

10. Genua, M. I., Miró, B., Hernanz, R., Miró, M., Pardo, C. Geriatría. En: Bonal J, Domínguez-Gil A, Gamundi M.C., Napal V., Valverde E. editores. Farmacia hospitalaria. Tomo $3.3^{\circ}$ ed. Madrid: Doyma; 2002; p. 959-991. Disponible en: https://www.sefh.es/bibliotecavirtual/fhto mo2/CAP08.pdf

11. Arriola Riestra I., Santos Marino J., Martínez Rodríguez N., Barona Dorado C., Martínez-González JM.
Consideraciones farmacodinámicas y farmacocinéticas en los tratamientos habituales del paciente gerodontológico. Av. Odontoestomatol. 2009; 25 (1): 29-34. Disponible en:

http://scielo.isciii.es/pdf/odonto/v25n1/ori ginal3.pdf

12. Malgor V. Farmacología geriátrica farmacocinética e interacciones entre drogas en geriatría. S.f. En Malgor, L.A., Valsecia, M.E. Farmacología Médica. Sf Vol 4. 169 p. 87-92. Disponible en: https://issuu.com/carlosquitanillarauch/d ocs/volumen iv/90

13. Miro, M., Borrull, A. Farmacocinética y Farmacodinámica. Atlas de medicina geriátrica en residencias. Editores Médicos, S.A. EDIMSA.

14. OMS. El incumplimiento del tratamiento prescrito para las enfermedades crónicas es un problema mundial de gran envergadura [Internet]. Ginebra; jul. 2003. [acceso en dic. 2020] Disponible en: https://www.who.int/mediacentre/news/re leases/2003/pr54/es/

15. Dillaa T, Valladaresa A, Lizánb L, Sacristán J. Adherencia y persistencia terapéutica: causas, consecuencias y estrategias de mejora. Aten Primaria. Jun. $2009,41(6): 342-346$. DOI: 10.1016/j.aprim.2008.09.031

\section{Correspondencia:}

rosmery_liz_2x@hotmail.com
Fecha de recepción: 06 de junio del 2020

Fecha de aceptación: 12 de noviembre del 2020 